\title{
CONSIDERING ECONOMIC NEEDS OF THE COMMUNITY OF PHOBJIKHA, BHUTAN: CONSTRAINTS AND CHALLENGES
}

\author{
LAM DORJI ${ }^{*}$ and JIGME TOBGAY ${ }^{2}$ \\ ${ }^{1}$ Executive Director, ${ }^{2}$ Coordinator - Conservation and Development Unit, Royal \\ Society for the Protection of Nature, Thimpu, Bhutan
}

Proceedings of the South and Central Asian MAB Meeting of Experts on Environmental Conservation, Management and Research, Hikkaduwa, Sri Lanka 15-18 October 2002

\begin{abstract}
Summary: A study conducted in the conservation area of Phobjikha (Wangdiphodrang, Bhutan) reveals that the local perceptions of the benefits emanating from conservation of Black-Necked Cranes is greater than their perceptions of associated costs. This is solely attributed to the moral and aesthetic value of cranes for their inherent magnificence and significance in local culture and traditions. No significant economic benefit was reported, while negative trends in viewing BNC conservation as an obstacle to development became evident. Community identified issues show the needs of the local people in terms of economic development. This led Bhutan's only national conservation NGO, the Royal Society for the Protection of Nature (RSPN), to initiate the Black-Necked Crane conservation programme in the area, based on the concept of the Integrated Conservation and Development Programme (ICDP). Plans and programmes to enhance conservation as a basis for local economic development were developed and are currently being implemented. Challenges and constraints to developing the area into a model conservation and development area have been identified. The challenges and constraints in implementing the so called ICDP are multi faceted, involving multiple actors such as government sector agencies, NGOs, local people consisting of farmers, business entrepreneurs, and religious bodies together with their own development, economic, conservation and religious objectives.
\end{abstract}

Heterogeneous community expectations for instant benefits at individual level, national policies, lack of local initiatives, low human resource capacity and ad-hoc programmes of different agencies are constraints that potentially slow programme implementation. The overarching causes are lack of awareness and the low econgmic standard of the local population that do not allow individuals to think beyond their own benefits. The challenge is for the human population in the area to gain a fairly good level of awareness about coordinated efforts that can enhance their participation in ongoing and future conservation and development programmes.

\section{Introduction}

Environmental conservation has emerged as an important topic for consideration in economic development of both developed and developing countries. At global, regional and national levels, the degrading quality of the environment is a concern of both the rich and the poor. Reactions to this concern come in the form of projects

\footnotetext{
" Corresponding author
} 
and programmes that are geared towards the conservation or restoration of the natural resources and ecosystem components. These projects and programmes are implemented with monetary assistance from the rich, with regulations and at best with support and participation of the local people. Some projects and programmes have succeeded while some have not. Successes and failures in most cases are judged from the external viewpoints which consider specific compartmentalized short-term objectives and often fail to consider the holistic local views. Viewpoints of local people help steer projects and programmes to long term success. The successful ones, in the modern sense, enhance economic development without compromising the quality of the environment or enhance the environmental quality of the ecosystem components. ${ }^{7,8}$

In this paper, we discuss the findings of a study ${ }^{2}$ of the aptitude value of conservation in Phobjikha (Wangdiphodrang, Bhutan); the experiences of the Royal Society for the Protection of Nature in its conservation initiatives, focusing on the constraints and challenges that need to be addressed in ongoing and future programmes in the area.

\section{Phobjikha Conservation Area: An Overview}

Phobjikha is a renowned winter habitat of the rare and endangered Black-Necked Cranes. Lying outside the northwestern tip of the Black Mountain National Park (now Jigme Singye Wangchuck National Park) and Phobjikha in the Wangdiphodrang district of Bhutan, it is situated at an altitude of 9,000 feet above sea level. Known for its scenic beauty, the area has a wide valley floor composed largely of marshes vegetated mainly with dwarf bamboo. Moderate to steep slopes comprising mainly of conifer forests and farmlands, encircle the valley. It represents a high altitude marshland ecosystem where nature and human beings are said to exist in harmony. The Nakay Chu meanders its way through the valley.

The Phobjikha soil is composed of quartzite and is conglomerated and has been classified by the Geological Survey of India as the Nakay Chu Formation. Geologically, there is evidence of glacial deposits projecting at the base of the Semchubara - Jangchey slope i.e., at the base of the hill where the Gangtey monastery stands.

The climate ranges from moderate summer to extreme winter, with temperatures ranging from $20^{\circ} \mathrm{C}$ in summer to as low as $-6^{\circ} \mathrm{C}$ in winter. Precipitation ranges from $50 \mathrm{~mm}$ to $300 \mathrm{~mm}$. July-August represent the warm and wet season and January is the coldest month.

More than three hundred households belonging to four administrative blocks (Geowgs) inhabit the Phobjikha valley. The settlements are mainly clustered and located along the valley sides. The valley is accessible by an unpaved road. There is a basic health unit (BHU) and a primary school. Almost all the people are engaged 
in subsistence farming, and potato is the main cash crop. Cattle, horse and sheep grazing helps to maintain the growth of bamboo to not more than $40 \mathrm{~cm}$. Many. households migrate to lower areas during winter.

The local people are Buddhists and their reverence for nature is noted. Local legend has it that Chu Karp, referred to as the wild boar, and Chu Nap, referred to as the snake, once had a bet. They were to see who first reaches the point of their present confluence. The bet was that if Chu Nap reached it first, Phobjikha would be capable of producing rice, and if Chu Karp reached it first, Phobjikha would not be capable of producing rice. Unfortunately, Chu Karp, the boar, rushed straight down to the point, while Chu Nap, the snake, was meandering his way through. That's the legendary reason as to why the Nakay Chu meanders its way through the valley and why paddy cannot be grown in Phobjikha.

\section{Significance of the Conservation Area}

The significance of Phobjikha is evident in its ecological characteristics as a beautiful high altitude marshland with faunal and floral diversity. It is particularly known for its status as a Black-Necked Crane habitat. In the northern part of the valley, the lower slope, adjacent to the valley floor, is dominated by blue pine (Pinus wallichiana), with hard woods such as birch (Betula utilis), and several species of rhododendron and maple (Acer spp.) in the understorey. Proceeding up the slope, the species composition changes to spruce-fir with suppressed hemlock (Tsuga dumosa) and rhododendron in the mid-storey. The areas of sparse canopy are dominated by hemlock. The understorey regeneration comprises of blue pine, dwarf bamboo (Arundinaria maling), Pteridium fern and herbaceous species such as Primula sp., Robus sp., and Fragaria sp. Forests in the southern part of the valley are richer in hardwoods dominated by birch, maple and rhododendron. The local fauna include wild boar (Sus scrofa), sambar deer (Cervus unicolor), muntjac (Muntiacus muntjac), Himalayan black bear (Selenarctos thibetamus), leopard (Panthera pardus), and red fox (Vulpes vulpes). There are over 62 species of birds in the area.

Above all, Phobjikha is a very important habitat of the rare and endangered Black-Necked Crane (Grus nigricollis). Of the fifteen species of cranes worldwide, the Black-Necked Crane (BNC) is one of the rare and endangered. The BNCs are migratory birds that come to winter in Bhutan. Bomdeling in TashiYangtse and Phobjikha (the study area) in the Wangdiphodrang districts are two important wintering grounds in Bhutan. Other wintering grounds in Bhutan include Tangmachu in Lhuntse and Gyetsa and Thangbi in the Bumthang district. Phobjikha hosts the largest group of these visiting birds and therefore is an important conservation area. 
Owing to their rare and endangered status, the BNCs have received the support of the Royal Government of Bhutan in securing legal protection. They are totally protected under Schedule I of the Forest and Nature Conservation Act of Bhutan 2002. Bhutan is a safe haven for wild animals and birds because of the country's pro environmental policy. The valleys of Phobjikha, Khotokha, Bomdeling and Bumthang are three places where BNCs spend their winter months. Of these places, Phobjikha and Bomdeling are the major habitats. Phobjikha has therefore been declared a conservation area for BNCs (BAP 1998, 2001). The survival of the $\mathrm{BNC}$ is a worldwide concern and in view of this concern, Phobjikha is not only a nationally important conservation area of the BNC but is also important globally. Recently, the importance of Phobjikha has become more evident with an increase in the member of cranes every year, in contrast to other areas which have reported a decline in the number of cranes. (Figure 1). Of the estimated $400 \mathrm{BNCs}$ that come to winter in Bhutan annually, Phobjikha is host to approximately 250 cranes.

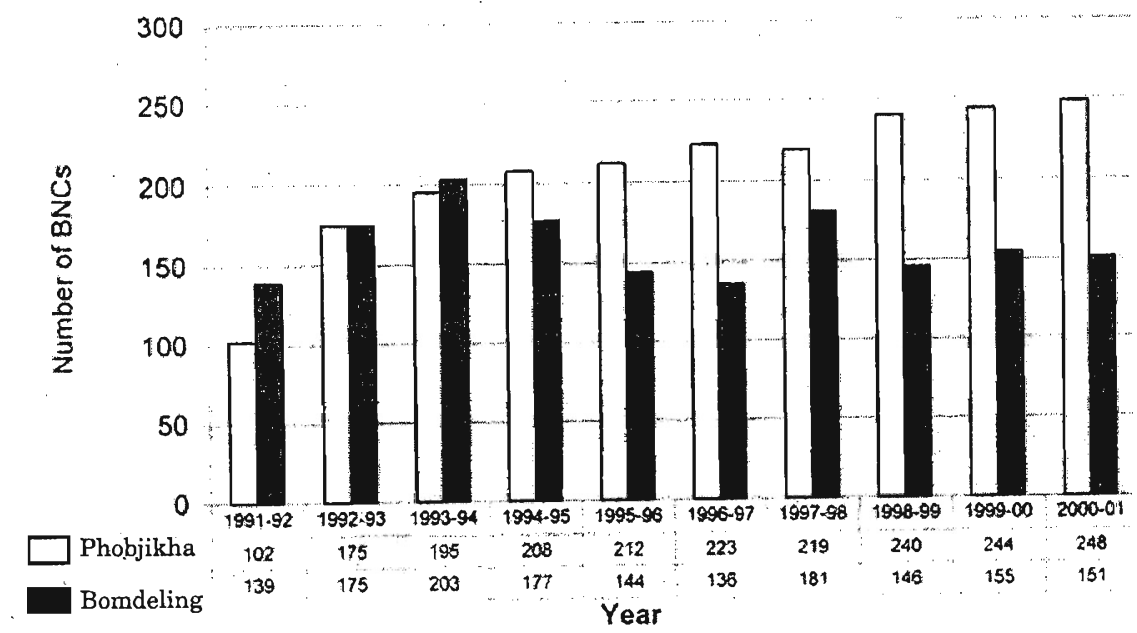

\section{Figure 1: Black-Necked Crane Population (1992 to 2001)}

The Royal Society for the Protection of Nature (RSPN), the only national non-profit making conservation NGO, was initially involved in the conservation of the BNCs in Bhutan. It merely engaged itself in monitoring and conducting annual counts of the cranes. Phobjikha's increasing importance as a conservation area, coupled with local peoples'needs and aspirations for development, increased RSPN's concerns for the future of BNCs in particular and the environmental condition of the valley in general. This concern led to initiations of RSPN programmes in Phobjikha. ${ }^{11}$

\section{Contingent Value of Conservation in Phobjikha}

Prior to 1998, conservation activities initiated in Phobjikha were merely in the form of regulations (on the part of the government) and annual counts conducted by the 
RSPN. The cranes, in addition to being protected by law, were also noted to have a very significant place in the local culture and tradition. It was also noted that there was some form of harmonious co-existence with nature. In 1998, as part of a master's thesis, a study was conducted to assess the value of conservation in Phobjikha.

The methods employed were based purely on the idea of contingent valuation. However, the study considered local people's perspectives and knowledge only, and any influence of external scientific reasoning about the value of conservation was avoided. 234 out of 312 households scattered over 38 isolated households/groups of households were interviewed.

Population and Local Economy: The study showed that the local population comprised of 43.2 per cent farmers, $9.5 \%$ students and $33 \%$ children between five and eighteen years of age and the rest were monks, military personnel, government servants etc. The local economy was one of purely subsistence with 86.6 per cent of the population depending on potato cultivation for their income. 2.6 per cent relied on government loans ( $13 \%$ interest rate) and $27.8 \%$ were exploited by local moneylenders at high interest rates of 20 to $25 \%$ per annum.

Issues in the valley: Although an area may be of immense environmental or conservation significance, local populations are always faced with issues that become challenges to organizations and agencies implementing projects and programmes in the area. In Phobjikha, there are a number of issues identified by the locals (Table 1). The list of issues and scores reflect local development needs and aspirations. Issues on unlicensed businesses existing in the valley (concerns raised by shopkeepers), bad road conditions, wildlife crop damage, lack of electricity, and unhygienic water supply are priorities that have a direct bearing on the economic welfare of the people.

Aptitude value of benefits - community perspective: The community perspective on the value of benefits arising out of crane conservation was measured. Using contingent valuation methods, the percentage benefits derived by the local people are grouped into five categories. Responses when inquired about the percentage benefit derived from various aspects of the conservation, are summarized in (Table 2).

The aptitude value of costs related to conservation of BNCs was also measured. Table 3 reveals that BNC conservation is not a total benefit but there are also drawbacks more pronounced in some villages rather than in others. There were times when a proposal for a new school had to be called off as the identified location fell in a habitat of the BNCs; there have also been times when the need to shift a existing school have been proposed. Furthermore; some villagers identified costs associated with damage to crops by the BNCs. Two villagers identified costs in terms of what they had to give up. The local people in Semchubara and Mal alleged that 
they were once allotted some land to be developed into pastures but that land was reclaimed on the ground that the pastures were going to affect the crane habitat. The non-existence of electricity in the valley has also been blamed on conservation.

Table 1: Issues Prioritized by Average Score

Issues

Frequency

Sum of Score

Av. Score

(Responses)

Road condition - bad

12

218

18.17

Water supply - unhygienic

9

116

Wildlife attack on crops

8

122

15.25

No electricity

8

121

15.13

No bridge during summer

6

99

16.50

Potato prices - risky

6

98

16.33

Schools - few teachers / too far

3

38

12.67

Weeds/ Blight or insect pests

2

33

16.50

No plots for separate livestock sheds

2

28

14.00

Pasture seed / fertilizers / fences

2

25

12.50

Unlicensed businessmen

1

20

20.00

Pasture plots - legal registration

1

15

15.00

Costly farm tools

1

15

Fuelwood shortage

1

12

12.00

Forestry - less staff / permits too far

3

32

10.67

Druk Seed Corporation - not beneficial 1

10

10.00

Unfavorable climate / soil

1

10

10.00

Cottage industries / handicrafts

19

9.00

Inferior livestock breed

3

22

7.33

Seeds not reliable for high altitude

2

13

The study concluded that the success of crane conservation was attributed to three important aspects.

- The pro- conservation policy of the Royal Government of Bhutan and the commitment to safeguard the habitat of the cranes as shown by declaring the area a conservation area. 
- Local cooperation in crane conservation as a result of the rich culture and tradition of the local people, based on the Buddhist ethics of revering other sentient beings, as well as owing to the fact that cranes are depicted in local folklore and tradition.

- The socioeconomic way of life of the people that has evolved to suit the natural limitations of the area (physical, extreme climatic conditions) make the area the perfect winter habitat of the cranes. During winter, human activities are limited due to extreme cold. Some people migrate to warmer areas, while cranes migrate into the valley.

Table 2: Aptitude Value of Benefits

\begin{tabular}{lccc}
\hline Benefit category & Frequency* $^{*}$ & $\begin{array}{c}\text { Av.Benefit } \\
(\%)\end{array}$ & Std. Deviation \\
\hline $\begin{array}{l}\text { BNC - a heavenly bird } \\
\text { (moral/aesthetic benefit) }\end{array}$ & 14 & 61 & 33 \\
$\begin{array}{l}\text { BNC brings development } \\
\text { (eg. Tourism) }\end{array}$ & 8 & 77 & 26 \\
$\begin{array}{l}\text { Sure benefit - but can't point out } \\
\text { (confidence) }\end{array}$ & 3 & 73 & 25 \\
$\begin{array}{l}\text { Conservation benefits } \\
\text { Enhanced sales }\end{array}$ & 1 & 85 & 0 \\
\hline
\end{tabular}

"Isolated households or groups of households under one name.

Table 3: Aptitude Value of Costs

\begin{tabular}{lcc}
\hline Cost aspect & Frequency & Av. Cost (\%) \\
\hline BNCs damage crops & 5 & 9 \\
Objections to pasture development & 2 & 18 \\
Need to shift existing schools & 1 & 55 \\
School at Tamshithang given up & 1 & 50 \\
Obstacle to the installation of electricity & 1 & 40 \\
\hline
\end{tabular}

Although the benefit-cost analysis showed that the local people as a whole perceived more benefits than costs emanating from crane conservation, two aspects became very clear:

- The benefits derived from the conservation of cranes were seen mainly in the aesthetic/ moral value of cranes. 
- There is a trend in people's attitudes whereby the costs of crane conservation are being seen in terms of what had to be given up (opportunity costs). People are becoming more conscious of the economic opportunities.

The study recommended an integrated approach to conservation in the area. Black-Necked Cranes could be conserved as an indicator species, which would automatically mean conservation of many other species and ecosystem components.

\section{Integrated Crane Conservation and Development Programme}

In line with the recommendations made, the Royal Society for the Protection of Nature initiated the Integrated Black-Necked Crane Conservation and Development Programme in 1999. The programme aimed at developing Phobjikha into a model that demonstrates conservation as a basis for human welfare and development, which in turn promotes conservation. Components of the programme include:

- The conservation component that encompasses conservation education; wetland restoration; research, monitoring and evaluation.

- The linking of conservation and development component that encompasses community organizations and capacity building, alternative energy, improvement of socioeconomic conditions, and eco-tourism development.

\section{Constraints and Challenges: Experiences of the RSPN}

Although the integrated conservation and development programme in Phobjikha is ongoing and therefore it is too early to talk about successes and failures, the experiences of the RSPN as an NGO involved in the area are worth noting. There are certain aspects in the area that hinder the efficient implementation of the programmes and pose enormous challenges to the organizations involved:

Heterogeneity: The Phobjikha Conservation area is heterogenous in two ways i.e. multiple administrative blocks and implementing agencies.

Multiple administrative blocks in the same area: The greatest challenge in realizing the programme objectives is the problem of enhancing the sustainability of community ownership and management of the area. The area involves three administrative blocks that makes collective action difficult. An overall Phobjikha Conservation Area Programme Committee (PCAPC) was formed with representatives from the administrative blocks and government sector agencies. The block representatives concentrate on seeking avenues that would benefit their respective blocks even if they were involved in an overall committee that is to look at the entire area as a whole. This makes decision-making and consensus 
building difficult. Community organizing becomes difficult despite efforts to bring them together as one.

Multiple implementing agencies, overlapping roles and low level of coordination: The relevant conservation agencies in the Phobjikha conservation area include the RSPN; beat office of the territorial division of the Forest Department, park warden's office of the Nature Conservation Division of the Forest Department; Agricultural Extensions offices. Uncoordinated planning which requires local people to expend much of their time on frequent meetings to discuss respective sector concerns, leads to people wasting their time and at times being unable to participate.

The PCAP committee members who come from these three administrative blocks tend to look at programme components in terms of benefits for respective blocks rather than view benefits for the area as a whole. Similarly, there are various agencies that implement programmes and activities independently, and avenues for possible coordination are hardly explored. This only means that people need to spend more time as required by different agencies, in order for the planning and implementation processes to be a success.

Misconceptions about plans and reality: Planning processes require local discussions and interactive meetings followed by project development, fundraising and implementation. Plans are future activities that may or may not take place depending on factors such as the availability of funds. However, people mistake plans as being definite and often have expectations for instant benefits. Furthermore, it is often impossible to deliver benefits at individual levels, though they may be forthcoming in the long run. Local people often fail to see overall benefits, which if they did would be incentives for collective action.

Expectations for instant benefit at block or individual level: Expectations for instant benefits at individual level is high. It is often difficult for local people to comprehend long-term benefits, and therefore they do not find enough incentive to participate in activities that may deliver benefits in the long run. Furthermore, individual input into a common purpose is rare, as individuals do not see any benefit arising directly from such efforts. The idea of deriving satisfaction out of doing voluntary work for a common cause is not feasible to rural subsistence farmers. Even elected committee members (who are not paid for being committee members) fail to perceive the benefits of their sacrifices to the community at large.

Lack of local initiatives: Till today, there are no records of local group or individual initiatives geared towards achieving project goals. It partially emanates from the national wage policy and more importantly the individual's priority in economic pursuits. Since initiatives are expected to be taken by the leaders/ 
representatives who are PCAP committee members, should they be paid (apart from what is being paid by the government)? If so, by whom and how much are questions that arise.

Low human resource capacity: The PCAP Committee is in its infancy. Members representing the local community are hardly exposed to contemporary decisionmaking processes. The concepts and skills of democratic decision-making, recording minutes, accounts keeping, etc. are lacking.

National policies: There are two aspects of national policies that constrain the implementation of ICDP in Phobjikha.

Vague definition of conservation area: The Ministry of Agriculture has declared a number of areas as conservation areas. Although Phobjikha is a conservation area, there is no detailed information specifying the extent and boundary of the conservation area, neither are there guidelines, rules and regulations outlining what is permissible and what is not permissible in the area. The management plan for the area is available only in the form of project activities of RSPN, which is highly constrained by the above limitations.

National tourism policy : Building eco-tourism programmes around the BNCs is one of the main programmes identified to facilitate conservation as a basis for economic development. The national tourism policy that requires tourists to channel fixed tariffs to be paid through the Department of Tourism is a constraint. Tour operators expect any tourism initiative in the country to have already been paid for under the fixed tariff. The crane festival organized to generate alternative income to the community experienced reluctance on the part of tour operators to pay fees and they therefore avoided promoting the tour. Sustenance of local ecotourism initiatives would not be feasible if the current notion persists that local tourism products (old or new programmes/services) are compensated for under the fixed tariff paid at the central level. While there is complete agreement that the benefits of tourism tariff comes to the people in the form of free education, health, and infrastructure, individuals and/or communities fail to see a direct link between payments made at the central level and local tourism products (eg. use of nature trails maintained by the local people) used by the tourists.

National wage policy: The national wage policy promulgates that no individual is to be engaged in unpaid work. Local people including leaders tend to quote this policy even when it comes to participating in project work that is expected to deliver benefits to the community. Therefore, participation involves payment. However, a $50 \%$ labor cost sharing has been incorporated.

The overarching causes are lack of awareness and the low economic standard of the local population which do not allow individuals to think beyond their own benefits. 
The challenge for the human population in the area is to secure a fairly good level of awareness about coordinated efforts that can enhance their participation in ongoing and future conservation and development programmes.

\section{The Way Ahead}

It is clear from the current situation that Phobjikha is growing in significance as a crane habitat. However, its carrying capacity (to support cranes) is unknown and difficult to estimate. ${ }^{6}$ The success or failure of conservation endeavors in the area will entail the measuring of achievements not only in terms of natural resource conditions but also in terms of economic welfare and resulting participation in sustainably managing the natural resources. In this context, the following points are important for future successes:

1 The legal aspects of the conservation area need to be specified. The boundaries need to be defined, along with rules and regulations.

2 The roles and responsibilities of implementing agencies need to be defined and coordination must be promoted so as to enhance supporting roles.

3 Short-term and medium/long-term programmes need to be identified and implemented accordingly. ${ }^{5}$

With these points being noted, the RSPN, with support from the Phobjikha Conservation Area Programme Committee has raised several concerns with the government and incorporated them into the plans. The RSPN aspires that Phobjikha become a role model area where its conservation significance serves as a basis for economic welfare of the local people who will then promote conservation, which, unlike the vicious cycle of poverty, is called the 'Mandala of harmonious coexistence with nature'.

\section{References}

1 Dixon J. A. \& Sherman P. B. (1990). Economics of Protected Areas, a look at benefits and costs.

2 Dorji L. (1998). Conservation and Development Strategies: Considering economic needs of the Community of Phobjikha, Bhutan. Master's Thesis No. SR98-2. AIT, Bangkok.

3 Field B. C. (1994). Environmental Economics: An Introduction. Mcgraw Hill Inc. 
4 Hunter C. \& Green H. (1995). Tourism and the Environment, A sustainable relationship.

5 Inoue M. (2001). Short Term Report - Wetland Conservation and Community Development. Royal Society for the Protection of Nature, Thimphu.

6 IUCN, UNEP and WWF (1991). Caring for the Earth, a Strategy for Sustainable Living. IUCN, UNEP and WWF Gland, Switzerland.

7 Royal Government of Bhutan (1992). The Middle Path, National Environment Strategy for Bhutan. National Environment Commission Secretariat, Thimphu.

8 Royal Government of Bhutan (1995). Forest and Nature Conservation Act of Bhutan. Ministry of Agriculture, Thimphu.

9 Royal Government of Bhutan (1998). Biodiversity Action Plan for Bhutan 1998. Ministry of Agriculture, Thimphu.

10 Royal Society for the Protection of Nature (2000). Longterm Plan Document. Thimphu.

11 Royal Society for the Protection of Nature (2001). Annual Report. Thimphu. 